\title{
Heterovalent doping of a 1D hybrid pseudo-perovskite: B site vacancy and short-range order
}

\author{
C. Pipitone', F. Giannici', A. Martorana' ${ }^{1}$ A. Guagliardi², G. García-Espejo ${ }^{3}$, N. Masciocchi ${ }^{3}$ \\ ${ }^{1}$ Dipartimento di Fisica e Chimica "Emilio Segrè", Università di Palermo, viale delle Scienze, 90128 Palermo, Italy; \\ ${ }^{2}$ Istituto di Cristallografia \& To.Sca.Lab., Consiglio Nazionale delle Ricerche, via Valleggio 11, 22100 Como, Italy; \\ ${ }^{3}$ Dipartimento di Scienza e Alta Tecnologia \& To.Sca.Lab., Università dell'Insubria, via Valleggio 11, 22100 Como, Italy \\ candida.pipitone@unipa.it
}

Hybrid halide perovskites are currently at the forefront of energy materials research for their appealing optical and electronic features, but applications in working devices are still hindered by chemical/structural stability. To enhance their properties, new hybrid compounds with a wide range of different organic cations have been proposed in the last years. The choice of bulky organic cations can reduce the dimensionality of the inorganic scaffold from $2 \mathrm{D}$ to $0 \mathrm{D}$. These lower-dimensional perovskites, best defined as pseudoperovskites, feature useful structural flexibility that can be further exploited to enhance the materials properties. We present here monodimensional hybrid iodide pseudo-perovskites, with $\mathrm{Pb}^{2+}$ and $\mathrm{Bi}^{3+}$ as $\mathrm{B}$ site cations, and $\left(\mathrm{CH}_{3}\right)_{3} \mathrm{SO}^{+}$(TMSO) in the A site. The $\mathrm{Pb}$ or $\mathrm{Bi}$ end members are isomorphic, and crystallize in the Pnma space group with wires of $\left[\mathrm{BX}_{6}\right]$ octahedra along the a direction. As shown in the figure, the chains are continuous for the $\mathrm{Pb}$ sample, or interrupted, for the Bi sample, with a B-site vacancy every third site to maintain charge balance. We prepared doped samples with general formula ((TMSO) $)_{3} \mathrm{~Pb}_{3 x} \mathrm{Bi}_{2(1-x)} I_{9}$ with complete miscibility between $(0 \leq x \leq 1)^{[1]}$. In the a direction, the structure is especially sensitive to $\mathrm{Bi}$ and cation vacancy (whose stoichiometry is (1-x) in the formula above) content. Interestingly, the XRD patterns of the samples with high Bi content (e.g. $x=0.33$ ) feature a peculiar broadening of $h k l$ peaks having non-zero $h$, while $0 k l$ peaks remain sharp (Figure 1). This broadening points out to a short-range order in the sequence of Pb-Bi-vacancy of the doped structure chains that can be successfully modeled using a stochastic matrix approach to model the $\mathrm{Pb} / \mathrm{Bi} / \mathrm{V}$ probability sequences and reconstruct the experimental XRD traces. The influence of bismuth doping on the optical properties is also significant: even a few \% loading of bismuth lowers the band gap by about $0.5 \mathrm{eV}$. Further characterization using X-ray spectroscopies (X-ray Raman scattering, XANES) to correlate the local electronic states to Bi content is underway. This work is a first insight into the effect of inorganic cation doping on short-range order and electronic properties of a 1D hybrid pseudo perovskite structure.
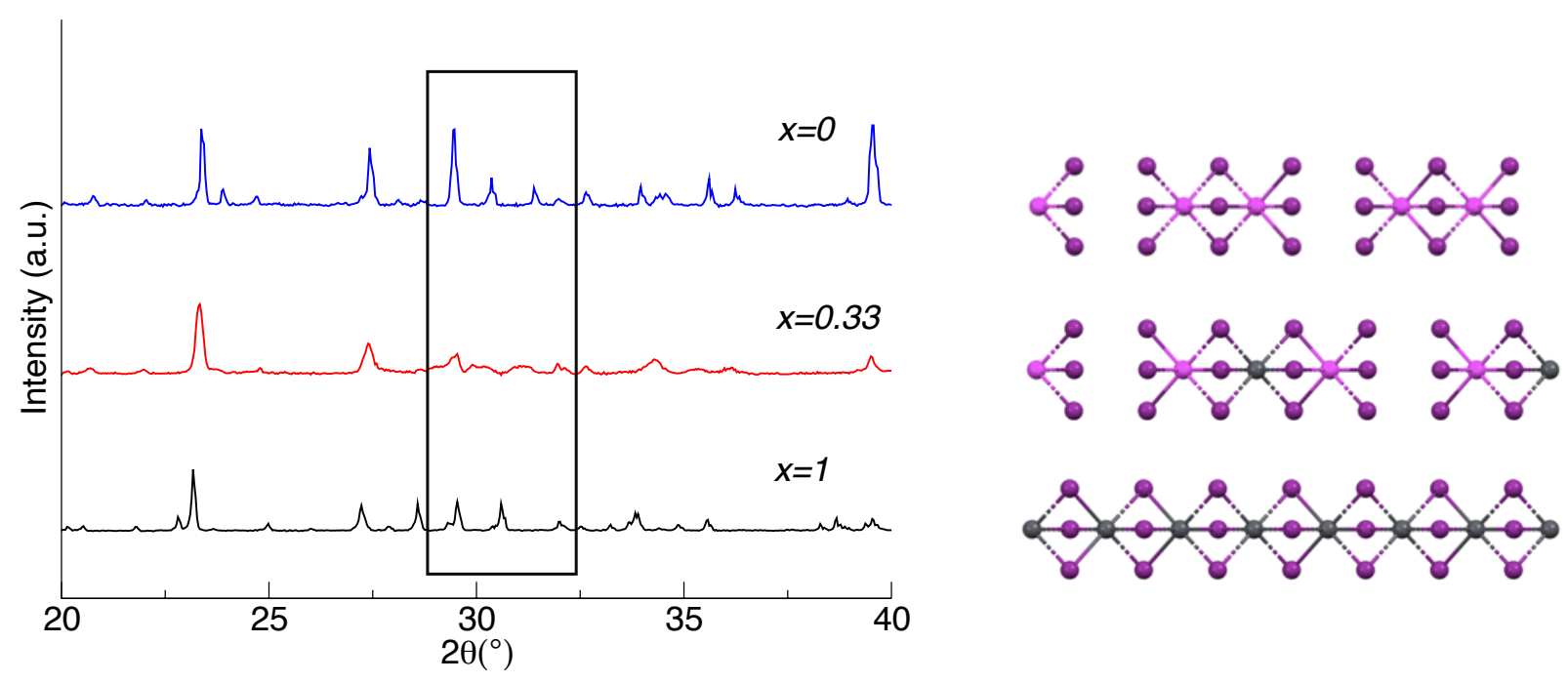

Figure 1 Magnification of XRD patterns of ((TMSO) $)_{3} \mathrm{~Pb}_{3 \times} \mathrm{Bi}_{2(1-\mathrm{x})} \mathrm{I}_{9}(x=1,0.33$ and 0$)$ and chains connectivity.

[1] C. Pipitone, F. Giannici, A. Martorana, S. Carlotto, M. Casarin, G. Garcìa-Espejo, A. Guagliardi, N. Masciocchi, J. Phys. Chem. C, in press.

Keywords: hybrid lead halides, trimethylsulfoxonium, powder diffraction, solid solution, ionic defectivity

This project was partially supported by MIUR (PRIN-2017L8WW48, Project HY-TEC). 